\title{
Maresin-1 reduces the pro-inflammatory response of bronchial epithelial cells to organic dust
}

Tara M Nordgren², Art J Heires², Todd A Wyatt ${ }^{1,2,3}$, Jill A Poole ${ }^{2}$, Tricia D LeVan ${ }^{1,2,4}$, D Roselyn Cerutis ${ }^{5}$ and Debra J Romberger ${ }^{1,2^{*}}$

\begin{abstract}
Background: Exposure to organic dust causes detrimental airway inflammation. Current preventative and therapeutic measures do not adequately treat resulting disease, necessitating novel therapeutic interventions. Recently identified mediators derived from polyunsaturated fatty acids exhibit anti-inflammatory and pro-resolving actions. We tested the potential of one of these mediators, maresin-1 (MaR1), in reducing organic dust-associated airway inflammation.
\end{abstract}

Methods: As bronchial epithelial cells (BECs) are pivotal in initiating organic dust-induced inflammation, we investigated the in vitro effects of MaR1 on a human BEC cell line (BEAS-2B). Cells were pretreated for 1 hour with 0-200 nM MaR1, followed by 1-24 hour treatment with 5\% hog confinement facility-derived organic dust extract (HDE). Alternatively, a mouse lung slice model was utilized in supportive cytokine studies. Supernatants were harvested and cytokine levels determined via enzyme-linked immunosorbent assays. Epithelial cell protein kinase $C$ (PKC) isoforms $a$ and $\varepsilon$, and PKA activities were assessed via radioactivity assays, and NFKB and MAPK-related signaling mechanisms were investigated using luciferase vector reporters.

Results: MaR1 dose-dependently reduced IL-6 and IL-8 production following HDE treatment of BECs. MaR1 also reduced HDE-stimulated cytokine release including TNF-a in a mouse lung slice model when given before or following HDE treatment. Previous studies have established that HDE sequentially activates epithelial PKCa and PKC $\varepsilon$ at 1 and 6 hours, respectively that regulated TNF- $\alpha, I L-6$, and IL-8 release. MaR1 pretreatment abrogated these HDE-induced PKC activities. Furthermore, HDE treatment over a 24-hour period revealed temporal increases in NFKB, AP-1, SP-1, and SRE DNA binding activities, using luciferase reporter assays. MaR1 pretreatment did not alter the activation of NFKB, AP-1, or SP-1, but did reduce the activation of DNA binding at SRE.

Conclusions: These observations indicate a role for MaR1 in attenuating the pro-inflammatory responses of BECs to organic dust extract, through a mechanism that does not appear to rely on reduced NFKB, AP-1, or SP-1-related signaling, but may be mediated partly through SRE-related signaling. These data offer insights for a novel mechanistic action of MaR1 in bronchial epithelial cells, and support future in vivo studies to test MaR1's utility in reducing the deleterious inflammatory effects of environmental dust exposures.

Keywords: Maresin-1, Pro-resolving mediators, Organic dust exposures, Airway inflammation, Bronchial epithelial cells

\footnotetext{
* Correspondence: dromberg@unmc.edu

${ }^{1}$ VA Nebraska-Western lowa Healthcare System, Research Service, Omaha, NE, USA

${ }^{2}$ Pulmonary, Critical Care, Sleep \& Allergy, University of Nebraska Medical

Center, Omaha, NE, USA

Full list of author information is available at the end of the article
}

\section{Biomed Central}

(c) 2013 Nordgren et al.; licensee BioMed Central Ltd. This is an Open Access article distributed under the terms of the Creative Commons Attribution License (http://creativecommons.org/licenses/by/2.0), which permits unrestricted use, distribution, and reproduction in any medium, provided the original work is properly cited. 


\section{Background}

Agricultural-related organic dust exposures are known to trigger airway inflammation. Individuals working within environments such as concentrated animal feeding operations (CAFOs) experience chronic respiratory diseases associated with this work [1,2]. Airway inflammation resulting from organic dust exposure is characterized by heightened pro-inflammatory cytokine release, neutrophil infiltration, and tissue remodeling processes. Long-term effects of chronic exposure include increased risk for lung function loss and obstructive pulmonary diseases [3]. Preventative measures such as the use of respirator masks to limit dust exposure are available, although these measures are not widely adopted or consistently utilized amongst the exposed populations [4]. Currently available therapeutics do not adequately treat or alleviate disease [5]. Therefore, improved preventative and therapeutic options are needed to assist this population of affected individuals.

To develop better-tailored therapies to prevent or treat organic dust-related airway inflammation, the basic biology underlying the disease must be more fully investigated. Previously published research using organic dust extracts derived from hog CAFOs (HDE) has shown a single exposure to organic dust causes bronchial epithelial cells (BECs) to release early response cytokines (tumor necrosis factor- $\alpha[\mathrm{TNF} \alpha]$, interleukin-6 [IL-6], and interleukin-8 [IL-8] in vitro or keratinocyte-derived chemokine [KC] and macrophage inflammatory protein-2 [MIP-2]) in vivo, leading to the recruitment of pro-inflammatory neutrophils and macrophages [6]. This pro-inflammatory HDE-stimulated cytokine release in BECs requires the activation of PKC $[7,8]$. Inhibiting the activation of pathways associated with these pro-inflammatory processes in BECs may alleviate the subsequent detrimental lung inflammation.

Specialized pro-resolving lipid mediators (SPM) such as resolvins, lipoxins, protectins, and maresins are derived from the metabolism of polyunsaturated fatty acids (PUFAs) [9-11]. These mediators have been shown to exhibit a variety of cell type-specific anti-inflammatory and pro-resolving effects, as reviewed by G. Bannenberg in 2010 [12]. These effects include reducing neutrophil infiltration, polarizing macrophages to an M2 phenotype while increasing phagocytic capacities, modulating proinflammatory cytokine release by epithelial cells and promoting neutrophil clearance across mucosal surfaces. While reducing inflammation, SPM have also been shown to increase lung immunity and resistance to infection $[13,14]$. These attributes make lipid mediators favorable candidates for treating pulmonary diseases characterized by neutrophil-emphasized inflammatory processes, such as those associated with organic dust exposures.

The potential utility of PUFAs or PUFA-derived mediators has not yet been studied in the context of organic dust exposures, although published literature reporting their use in other similar models of inflammation has demonstrated a potential therapeutic application. For example, resolvin E1 has shown promise in reducing proinflammatory cytokine release, improving host immunity in the context of acute lung injury, and reducing cell infiltration and airway hyper-responsiveness in a murine model of asthma [14-16]. Resolvin D1 has been reported to promote resolution of airway inflammation induced by cigarette smoke as well as acute lung injury caused by LPS in mice $[16,17]$. Although not yet studied in the lung environment, MaR1 has shown utility in reducing neutrophil infiltration while increasing macrophage phagocytic capacities in a murine model of peritonitis [18]. Previously published data from our group suggest the recruitment and subsequent actions of macrophages in organic dust exposures are highly important in determining the outcomes of the pro-inflammatory insult, and pro-inflammatory cytokine production by BECs exposed to injurious stimuli such as organic dusts is key to the recruitment of these macrophages as well as neutrophils into the lung [19-23]. However, MaR1's effects on BECs, along with other cells in the lung are currently unknown.

In consideration of the important roles that BECs play in potentiating the pro-inflammatory effects of HDE, including the release of cytokines, in part through protein kinase $\mathrm{C}$ (PKC) isoform activation, and recruitment and activation of other leukocyte responders [7,24-26], the purpose of our study was to determine whether MaR1 would reduce the pro-inflammatory effects in BECs induced by HDE. Endpoint measurements included PKC $\alpha$ and PKCe activities, transcription factor binding activities and cytokine release. Results of the experiments reported here demonstrate that MaR1 can reduce dust-induced PKC $\alpha$ and $\mathrm{PKC} \varepsilon$ activation and pro-inflammatory cytokine release in the BECs. While $\mathrm{NFKB}, \mathrm{AP}-1$, and SP-1-related signaling are important to the pro-inflammatory response of BECs to HDE, MaR1 pretreatment did not reduce the DNA-binding activities of these transcription factors. Although, the HDE-induced DNA binding activities at the serum response element (SRE) is reduced upon MaR1 pretreatment, suggesting this pathway is modified by MaR1. Taken together, these data reveal previously uncharacterized anti-inflammatory effects of MaR1 on BECs and ex vivo mouse lung slice cultures exposed to HDE and support future in vivo studies testing the utility of MaR1 for potential treatment of organic dustmediated lung inflammation.

\section{Methods}

Materials

7(S)-Maresin-1 (7S,14R-dihydroxy-4Z,8E,10Z,12Z,16Z, 19Z-docosahexaenoic acid) and 7(R)-Maresin-1 (7R,14Sdihydroxy-4Z,8E,10E,12Z,16Z,19Z-docosahexaenoic acid) were obtained from Cayman Chemical (Ann Arbor, MI, USA). The human bronchial epithelial cell line BEAS-2B 
was purchased from American Type Culture Collection (Manassas, VA, USA).

\section{Animal care and housing}

Male $\mathrm{C} 57 \mathrm{Bl} / 6$ mice were purchased from Jackson Laboratories (Bar Harbor, ME, USA) and housed in cages (group housing) under pathogen-free conditions. Mice received a standard mouse chow diet, and care was supervised by the University of Nebraska Medical Center Animal Care Facilities. Experimental use of animals was regulated and approved by the University of Nebraska Medical Center Institutional Animal Care and Use committee.

\section{Preparation of mouse lung slices}

Mouse lung slices were prepared using previously described methodology $[27,28]$. Briefly, C57BL/6 male mice were euthanized with $50 \mathrm{mg} / \mathrm{mL}$ pentobarbital, and lungs filled with low melting point agarose. Lungs were sliced using a vibrating microtome (EMS-4000; Electron Microscope Sciences, Hatfield, PA) and cultured for 4 days in RPMI medium (with 2 medium changes) prior to use in experiments.

\section{Tissue culture}

BEAS-2B cells were grown as submerged cultures in serum-free LHC-9 (Invitrogen; Grand Island, NY):RPMI (Sigma; St. Louis, MO, USA) media (1:1) containing 100 $\mathrm{U} / \mathrm{ml}$ Penicillin $+100 \mu \mathrm{g} / \mathrm{ml}$ Streptomycin (Invitrogen; Grand Island, NY, USA). Cells were incubated at $37^{\circ} \mathrm{C} / 5 \%$ $\mathrm{CO}_{2}$ and passaged via trypsinization. Experiments were performed using cells of approximately $85 \%$ confluency.

\section{Preparation of organic dust extract}

Organic dust extract was prepared as previously described [7]. Briefly, settled dust from hog confinement facilities was placed in Hanks' balanced salt solution (Biofluids; Rockville, MD, USA) (1 gram dust per $10 \mathrm{ml}$ solution). This solution was incubated for 1 hour, followed by two vortexing and centrifugation steps. The resulting supernatant was sterilefiltered (0.2 $\mu \mathrm{M}$ filter) (Nalgene; Rochester, NY, USA) and aliquoted at $-20^{\circ} \mathrm{C}$.

\section{TNF-a, IL-6, and IL-8/CXCL1 cytokine levels}

BEAS-2B cells were pretreated for 1 hour with 0-200 nM $7(\mathrm{~S})$-MaR1 or $7(\mathrm{R})$-MaR1, followed by $5 \%$ HDE for 24 hours. Cell supernatants were collected and IL-6 and IL-8 enzyme-linked immunosorbent assays (ELISAs) were performed as previously described [7]. Alternatively, mouse lung slices were pre-treated for 1 hour with $0-200 \mathrm{nM}$ MaR1, followed by $5 \% \mathrm{HDE}$ treatment, or given $5 \% \mathrm{HDE}$ treatment followed by $0-200 \mathrm{nM} 7(\mathrm{~S})$-MaR1 $1 \mathrm{hr}$ after the HDE treatment was given. At 24 hours following HDE treatment, lung slice supernatants were collected and assayed for murine TNF- $\alpha$, IL- 6 , and the murine IL- 8 cognate CXCL1 using ELISAs.

\section{Transcription factor binding activities}

Cells were reverse transfected onto 96-well plates using Cignal Vector Reporters for NFkB, AP-1, SP-1, and SRE (SABiosciences; Valencia, CA, USA), using manufacturer's directions with Lipofectamine 2000 (Invitrogen; Grand Island, NY, USA). Transfected cells were treated with 5\% HDE (with or without 0-200 nM 7(S)-MaR1 pretreatment) for 1-24 hours, then harvested using Promega Dual-Glo Luciferase Reagent (Promega; Madison, WI, USA); luciferase activity was measured on a Victor $3 \mathrm{~V}$ plate reader (Perkin Elmer; Waltham, MA, USA). The luciferase vectors work such that a firefly luciferase reading that is inducible (i.e. AP-1 reporter) is obtained, as well as a constitutive renilla luciferase reading, for normalization. Normalized data is thus expressed as relative luciferase units, which are compared across treatment groups and expressed as fold-change values over controls.

\section{PKCa, PKC $\varepsilon$, and PKA activities}

BEAS-2B cells were pre-treated for 1 hour with 0,100 , or $200 \mathrm{nM} 7(\mathrm{~S})$-MaR1, followed by 1 hour or 6 hours treatment with 5\% HDE. Cells were lysed and measured for $\mathrm{PKC} \alpha$ and $\mathrm{PKC} \varepsilon$ kinase activities, as previously described [29-31].

\section{Statistical analyses}

Student's $t$ tests and ANOVA (Tukey's method for post-hoc multiple comparisons) tests were used to compare control versus treated groups, as appropriate. $\mathrm{P}$ values $\leq 0.05$ were considered significant. Data are expressed as mean +/standard error of the mean (SEM). Graphing/statistical analyses were performed using the Graphpad Prism software program.

\section{Results}

MaR1 reduces the release of HDE-induced proinflammatory cytokines (IL-6 and IL-8) by airway epithelial cells (BEAS-2B cell line)

Previous studies have shown treatment of BECs with 5\% HDE for 24 hours leads to significant increases in IL-6 and IL- 8 release $[7,8]$. To test the effects of MaR1 pretreatment on cytokine release from BECs in response to HDE, BEAS-2B cells were pretreated with $0,1,10,100$, or $200 \mathrm{nM}$ MaR1 (in all studies, the 7[S] form of MaR1 was used, unless otherwise stated). After $1 \mathrm{hr}, 5 \%$ HDE was added to the cultures and allowed to incubate for 24 hours. In cells receiving MaR1 pretreatment, IL-6 and IL-8 cytokine levels were dose-dependently inhibited compared to cells receiving HDE alone, as measured at 24 hours post-HDE exposure (Figure 1). In the absence of HDE, MaR1 alone had no effect on IL-6 or IL-8 levels 
at any concentration tested. These studies were repeated using the $7(\mathrm{R})$ isoform of MaR1, achieving similar results (data not shown).

MaR1, given prior to or during HDE treatment, reduces the release of pro-inflammatory cytokines (TNF-a, IL-6, and $(X C L 1)$ in a mouse lung slice model of HDE exposure To ascertain the reproducibility of MaR1's effects in a translationally relevant airway model system, mouse lung slices were used to test the capacity of MaR1 to limit HDE-induced cytokine release. Using the mouse lung slice model, precision cut mouse lungs slices prepared from C57Bl/6 mice were pretreated for 1 hour with 0,100 , or $200 \mathrm{nM} \mathrm{MaR} 1$ followed by 5\% HDE. Alternatively, lung slices were first treated with 5\% HDE, then given 0, 100, or $200 \mathrm{nM}$ MaR1concomitantly with HDE treatment (at 1 hour following treatment with HDE). At 24 hours following HDE treatment, TNF- $\alpha$, IL-6, and CXCL1 (murine IL-8 homolog) cytokine release were significantly inhibited in a dose-dependent manner in MaR1 pretreatment (Figure 2) and MaR1 post-treatment (Figure 3) studies. Taken together, these results show the ability of MaR1 to attenuate the release of the pro-inflammatory HDE-induced TNF- $\alpha$, IL-6, and CXCL1 cytokines as both a pretreatment as well as after initial HDE exposure (MaR1 given after introduction of HDE). Additionally, these results provide validation for the effects of MaR1 on HDE-stimulated cytokine release by BEAS-2B in culture.

\section{Effects of HDE with and without MaR1 pretreatment on $\mathrm{PKCa}, \mathrm{PKC} \varepsilon$, and PKA kinase activities}

Data from our group has shown HDE augments the release of TNF- $\alpha$, IL-6, and IL-8 from cultured airway epithelial cells and in vivo in a manner that is dependent, at least in part, on PKC activation [8]. Specifically, sequential activation of $\mathrm{PKC} \alpha$ and $\mathrm{PKC \varepsilon}$ isoforms occurs following HDE exposure, with PKC $\alpha$ activity peaking at 1 hour and
$\mathrm{PKC} \varepsilon$ activity peaking at 6 hours following HDE exposure $[7,8]$. In dominant-negative PKC $\alpha$ BECs, TNF- $\alpha$ is not produced, and $\mathrm{PKC} \varepsilon$ activation, IL-6, and IL- 8 release are abrogated. However, in PKCe dominant-negative BECs, TNF- $\alpha$ and IL- 6 release are not affected, but IL- 8 release is diminished [8]. These studies highlight the importance of PKC activation in the release of TNF- $\alpha$, IL- 6 , and IL- 8 in HDE-stimulated BECs. Therefore, to determine the effects of MaR1 on kinases that regulate the HDE-induced proinflammatory responses, BEAS-2B cells were pretreated with 0,100 , or $200 \mathrm{nM} \mathrm{MaR} 1$ for 1 hour, followed by 1 or 6 hour treatment with 5\% HDE. Cell lysates were then assayed for $\mathrm{PKC} \alpha$ and $\mathrm{PKC \varepsilon}$ kinase activity levels. As expected, in cells treated with 5\% HDE, PKC $\alpha$ levels were significantly increased within 1 hour following HDE exposure. Pretreatment with 100 or $200 \mathrm{nM}$ MaR1 eliminated this effect (Figure 4A). Similarly, in BEAS-2B cells treated for 6 hours with 5\% HDE, PKC $\varepsilon$ kinase activity was significantly stimulated over untreated controls, while maresin-1 pretreatment eliminated this effect (Figure 4B). MaR1 did not inhibit phorbol ester (PMA)-stimulated PKC $\alpha$ or PKC $\varepsilon$, suggesting that MaR1 is not functioning as a non-specific kinase inhibitor of these enzymes (data not shown). These results indicate MaR1 can reduce the activation of PKC $\alpha$ and PKCe during HDE stimulation of BECs. Because PKC activity is known to potentiate the proinflammatory responses in BECs exposed to HDE, MaR1's mechanism of reducing inflammatory cytokine release is likely mediated in part through these inhibitory actions on PKC $\alpha$ and PKC $\varepsilon$.

Previous experiments by our laboratory have shown that PKA activation negatively regulates the pro-inflammatory cytokine release in BECs induced by HDE exposure [32]. We therefore sought to determine whether or not MaR1 was inhibiting the release of pro-inflammatory cytokines not only by inhibiting PKC $\alpha$ and PKCe activity, but also through the activation of PKA. To determine the effects
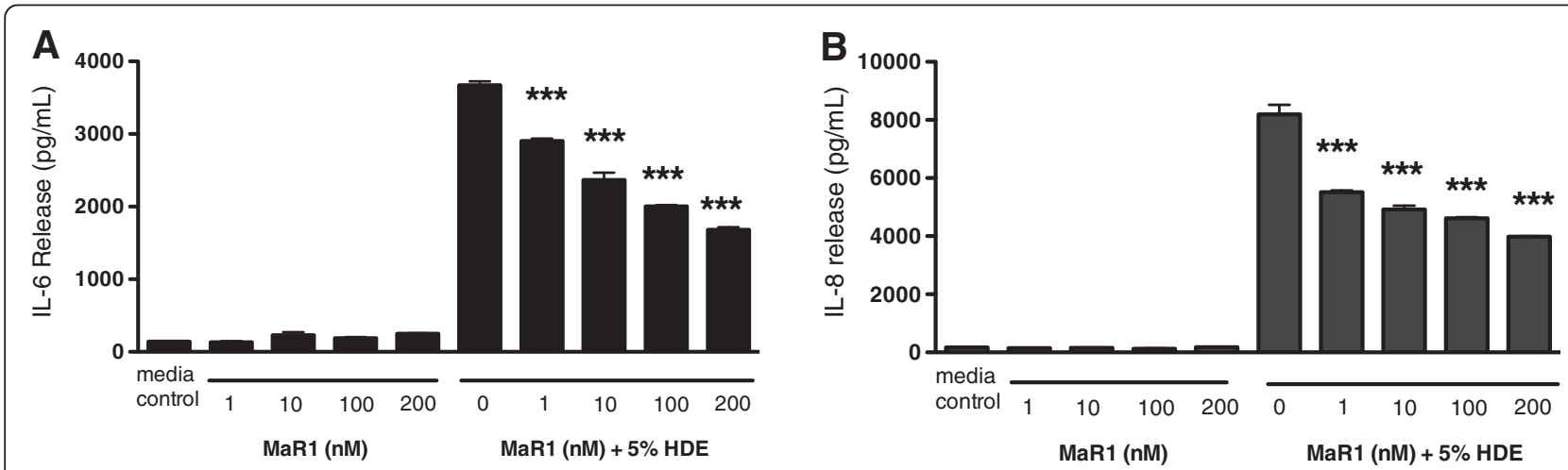

Figure 1 Effects of MaR1 pretreatment on HDE-induced IL- 6 and IL-8 release 24 hours following HDE treatment in bronchial epithelial cells. BEAS-2B cells were pretreated with $0,1,10,100$, or $200 \mathrm{nM} \mathrm{MaR1}$ for 1 hour, then treated with 5\% HDE. At 24 hours post HDE treatment, cell supernatant was collected and assayed for A) IL-6 and B) IL-8 levels, using ELISAs. ${ }^{* * *} \mathrm{p}<0.001$ compared to 5\% HDE. Data is representative of 4 separate experimental replicates (biological replicates), with two technical replicates per experiment. 
Figure 2 Effects of MaR1 pretreatment on HDE-induced TNF-a, IL-6, and CXCL1 release 24 hours following HDE treatment in mouse lung slices. Mouse lungs slices were pre-treated for 1 hour with 0,100 , or $200 \mathrm{nM} \mathrm{MaR1}$, followed by $5 \%$ HDE treatment. At 24 hours following HDE treatment, lung slice supernatants were collected and assayed for murine TNF-a (A), IL-6 (B), and the murine IL-8 cognate CXCL1 (C). ${ }^{* *} p<0.01$ vs. $5 \% \mathrm{HDE}^{*}{ }^{* * *} \mathrm{p}<0.001$ vs. $5 \%$ HDE. Data are representative of 4 separate experimental replicates.

of MaR1 on PKA, we pretreated BEAS-2B cells with 0 , 100 , or $200 \mathrm{nM} \mathrm{MaR} 1$ for 1 hour, then treated with $5 \%$ HDE for 1 hour. Cell lysates were then assayed for PKA activity. We found that MaR1 did not significantly alter PKA activity levels when given as a pretreatment to HDE for 1 hour (Figure 4C). Similarly, no change in PKA activity was observed after 6 hour HDE exposure in MaR1-pretreated cells (data not shown). These data suggest that the inhibition of PKC $\alpha$ and $\mathrm{PKC} \varepsilon$ and concomitant reduction in IL- 6 and IL- 8 release by BECs following HDE stimulation by MaR1 is independent of the actions of PKA.

\section{Effect of HDE on transcription factor binding activities in BECs with and without MaR1 pretreatment}

To determine the effects of HDE on downstream proinflammatory signal activation in BECs, Cignal Vector Reporters were used. Following reverse transfection of BEAS-2B cells with the luciferase vectors, cells were pre-treated with 0 or $200 \mathrm{nM} \mathrm{MaR} 1$ for 1 hour prior to stimulation with HDE. After 1 hour pretreatment, cells were challenged with or without $5 \% \mathrm{HDE}$ for $1,6,12$, or 24 hours. In HDE-treated cells, NFKB, AP-1, SP-1, and SRE transcription factor binding activities exhibited significant increases over control cells that received no HDE treatment in a time-dependent manner. No significant changes were seen in the binding activities of $\mathrm{NFKB}_{\mathrm{K}}$, AP-1, or SP-1 in cells that were given MaR1 pretreatment prior to HDE stimulation (Figure 5A-C). Although, at 24 hours following HDE exposure, there was a significant decrease in SRE activation in MaR1 pretreated cells (Figure 5D). These results suggest that the mechanism by which MaR1 modulates the pro-inflammatory responses of HDE-stimulated BECs is likely not propagated through $\mathrm{NF \kappa}, \mathrm{AP}-1$, and $\mathrm{SP}-1$ transcription factor-related signaling, but may be affecting the activation of SRE-related signaling mechanisms.

\section{Discussion}

Through these investigations, we have found a potential role for the SPM MaR1 in reducing the pro-inflammatory responses of BECs to HDE. These findings include reduced pro-inflammatory cytokine production and $\mathrm{PKC} \alpha / \varepsilon$ activities. In addition, studies performed using a mouse lung slice model indicate the utility of MaR1 in reducing 
Figure 3 Effects of MaR1 treatment given during HDE exposure on HDE-induced TNF- $a$, IL-6, and CXCL1 release 24 hours following HDE treatment in mouse lung slices. Mouse lungs slices were given $5 \% \mathrm{HDE}$ treatment. At 1 hour following the addition of HDE, 0, 100, or $200 \mathrm{nM}$ MaR1 was added to the slices. At 24 hours following HDE treatment, lung slice supernatants were collected and assayed for murine TNF-a (A), IL-6 (B), and the murine IL-8 cognate CXCL1 (C). ${ }^{*} \mathrm{p}<0.05$ vs. $5 \% \mathrm{HDE}^{*}{ }^{* *} \mathrm{p}<0.01$ vs. $5 \% \mathrm{HDE}$. Data are representative of 3 separate experimental replicates.

the pro-inflammatory cytokine release caused by HDE in a more complex and biologically relevant model system, where structural and lung resident cells remain in contact, simulating a more realistic biological environment than cell line cultures. Importantly, in the mouse lung slice model, MaR1 was shown to be effective at reducing pro-inflammatory cytokine release in mouse lung slices not only when given as pre-treatment to HDE, but when given 1 hour following HDE exposure as well. These data suggest the potential utility of MaR1 as a preventative as well as a therapeutic treatment in the prevention of airway inflammatory disease.

These findings are of particular importance, as organic dust-related airway inflammation causes multiple deleterious effects. Individuals who experience acute exposures to organic dust develop heightened neutrophilia and pro-inflammatory cytokine production in their airways. Prior studies indicate that BECs play an important role in releasing pro-inflammatory cytokines, which recruit inflammatory leukocytes into the lung following organic dust exposures. Therefore, preventative and treatment measures that reduce the stimulation of these cells by organic dust might prove clinically beneficial to patients. MaR1 may represent a novel class of drugs for preventing and/or treating the airway inflammatory consequences following organic dust exposures.

Recently discovered pro-resolving lipid mediators, such as the resolvins, protectins, lipoxins, and maresins have been found to have potent anti-inflammatory and pro-resolution effects on multiple different cell types that are relevant in the lung environment. For example, resolvin E1 can decrease the levels of IL- 6 and IL-1 $\beta$ found in lung tissue following $\mathrm{HCl}$-induced acute lung injury [14]. Resolvin E1 also limited lymphocyte recruitment, IL-13 release, and airway hyper-responsiveness in a murine model of asthma [15]. LPS-induced and cigarette smoke-induced lung inflammation was diminished by treatment with resolvin D1 $[16,17]$. The role of MaR1 has not yet been characterized within the lung, although studies investigating the effect of MaR1 in limiting inflammation in a murine peritonitis model highlight its potential in attenuating macrophage and neutrophil-dominating inflammatory diseases [18]. Our 


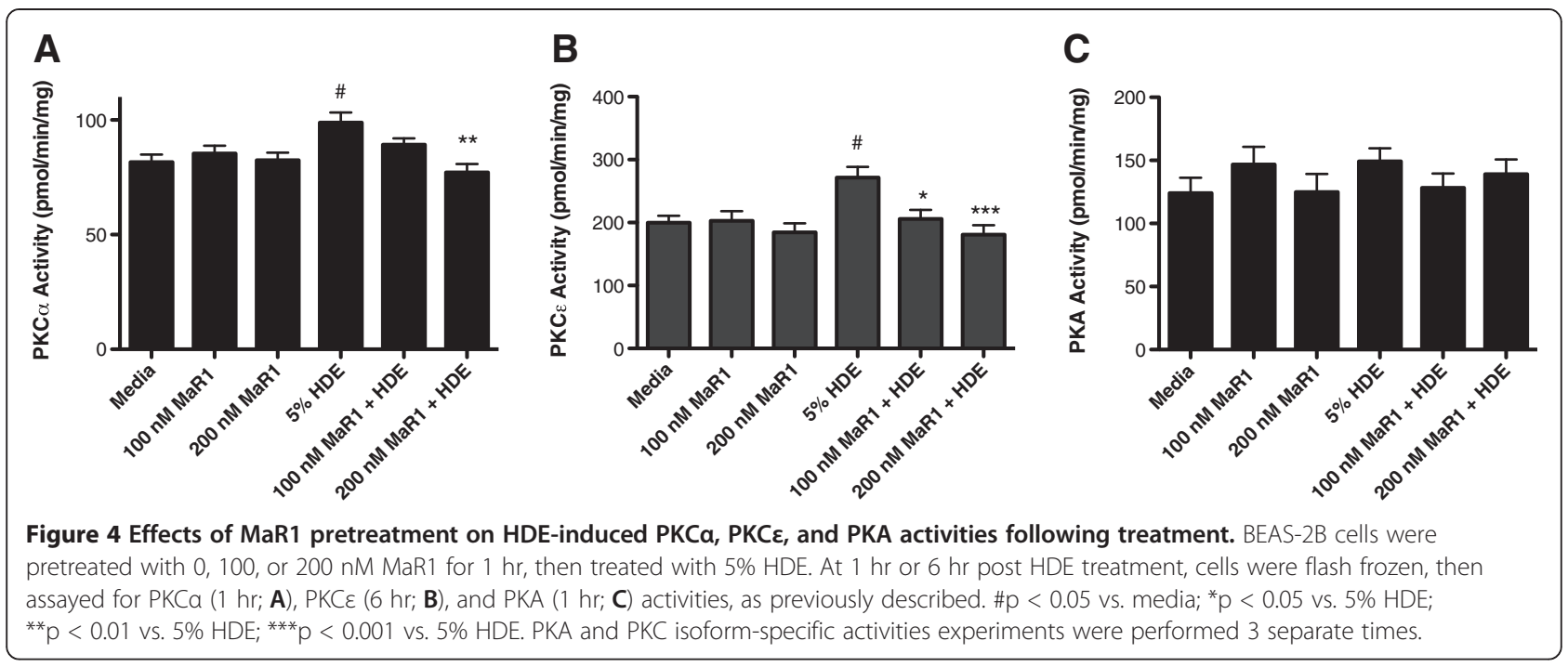

findings of reduced inflammatory cell-recruiting TNF- $\alpha$, IL-6, and IL-8/CXCL1 release in vitro (BEAS-2B cell line) and/or ex vivo (mouse lung slice model) suggests a potential anti-inflammatory role for MaR1 by reducing macrophage and neutrophilic-dominant organic dustinduced lung disease. In our studies, we chose to use concentrations of MaR1 ranging from $1-200 \mathrm{nM}$ based upon previous studies with MaR1 and other SPM $[18,33]$, and we found significant effects with as little as $1 \mathrm{nM}$ MaR1. These data suggest that MaR1 can effectively attenuate the HDE-stimulated pro-inflammatory cytokine release in BECs.
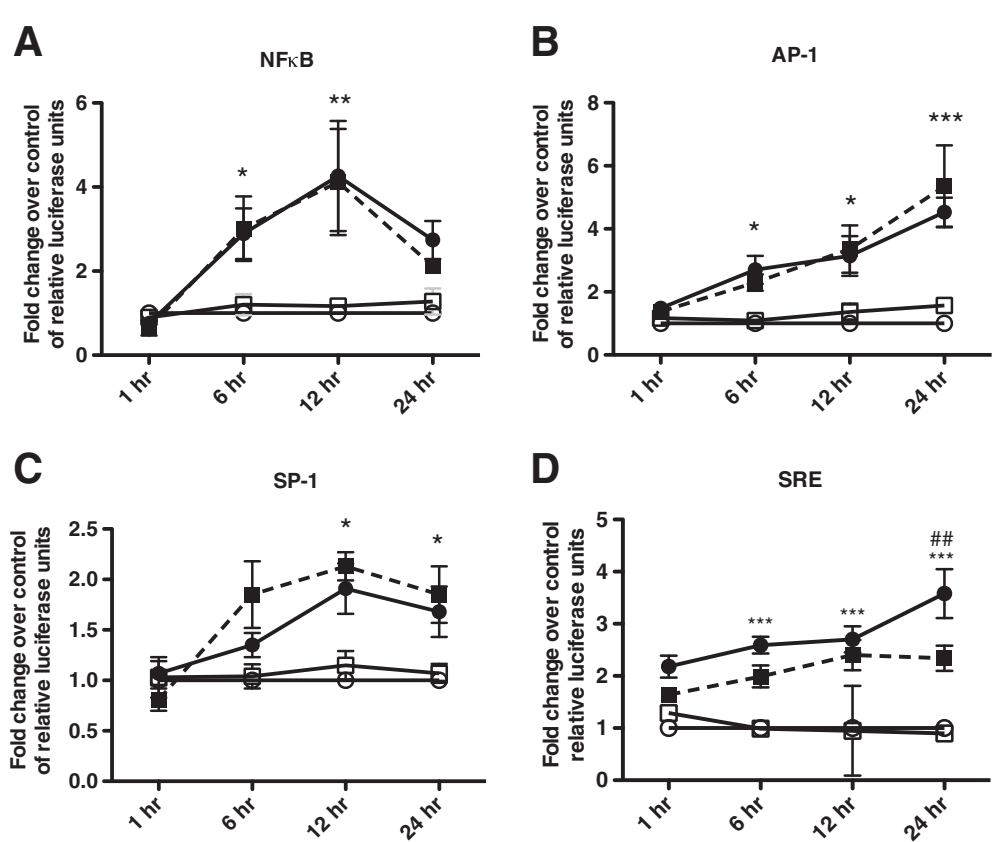

D

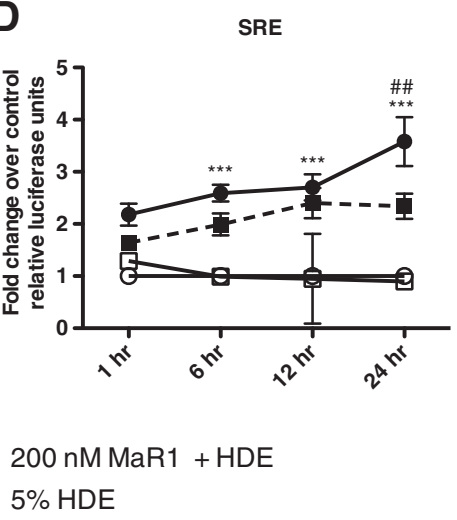

Figure 5 Effects of HDE on transcription factor binding activity in bronchial epithelial cells. BEAS-2B cells were treated with 0 or $200 \mathrm{nM}$ MaR1 for 1 hr, then treated with 5\% HDE. At 1, 6, 12, and 24 hours following HDE treatment, cells were harvested and assayed for NFKB (A), AP-1 (B), SP-1 (C), and SRE (D) transcription factor binding activities using SABiosciences dual luciferase vector systems. ${ }^{*} p<0.05$ vs. media; ${ }^{* *} p<0.01$ vs. media; *** $p<0.001$ vs. media. \#\# $p<0.01$ vs. HDE. Data are shown as a fold change of relative luciferase activity over media controls. Each time point was performed in triplicate at minimum. 
To better understand the mechanisms underlying the reduced inflammatory cytokine response following organic dust-exposed BECs and mouse lung slices with MaR1 pretreatment, a series of experiments were performed to analyze intracellular signaling events. We have previously reported that HDE-stimulated TNF- $\alpha$, IL-6, and IL- 8 release in BECs is dependent upon the sequential activation of $\mathrm{PKC}$ isoforms, where $\mathrm{PKC} \alpha$ is responsible for TNF- $\alpha$ release, and TNF- $\alpha$ leads to IL- 6 release and PKC $\varepsilon$ activation, while PKC $\varepsilon$ activation subsequently induces IL-8 release $[7,8]$. A summary of these previously published findings is provided in Figure 6. In this study, pretreatment with MaR1 prior to HDE stimulation effectively abrogated the early (1 hour) activation of PKC $\alpha$ and subsequent TNF- $\alpha$ release, IL- 6 release, and activation of PKC $\varepsilon$ at 6 hours. Although PKA activation has been implicated in inhibiting organic dust-induced PKC activation in epithelial cells [32], we observed no change in PKA activity in response to MaR1 pretreatment. Our data suggest that MaR1 interferes with HDE-mediated PKC activation indirectly, as MaR1 appeared to have no enzyme inhibitory effect itself in the presence of the direct PKC activator, PMA. We hypothesize that MaR1 action is upstream of PKC $\alpha$, potentially at the level of cell surface receptor signaling. As reviewed by Serhan, et al. in 2011, other PRM have been shown to bind specific g-protein coupled receptors to propagate agonist and antagonist effects, and this is a well-known mechanism of action for other poly-unsaturated fatty acid-derived lipid mediators (such as the prostaglandins) [34]. This therefore is likely how MaR1 also acts, although cognate receptors for MaR1 are currently undefined.

Additional potential intracellular signaling/transcriptional pathways were explored to explain the action of MaR1 in reducing pro-inflammatory cytokine release following HDE treatment in BECs. HDE treatment alone temporally activated the binding activities at the $N_{k} B$, AP-1, SP-1, and SRE DNA binding elements. Our laboratory and others have previously shown the importance of NFkB and MAPK-related pathway activation in various cells (i.e. phagocytes, epithelial cells) following organic dust exposures [37-40]. MaR1 pretreatment did not significantly affect the total binding activities of NFkB, AP-1, or SP-1. However, MaR1 did attenuate HDE-induced SRE binding activities at 24 hours following HDE exposure. Binding at SRE is known to be activated through both MAPK and Rho kinase signaling mechanisms via serum response factor (SRF) binding at SRE sites $[41,42]$. This reveals potential pathways that may be targeted by MaR1 to reduce HDE-induced proinflammatory consequences to BECs. Interestingly, the activation of SRF-related signaling and SRE binding activity has been shown to be dependent on PKC $\alpha$ and PKC $\varepsilon$ activities [42], corroborating with our findings that these enzymes are required for HDE-related pro-inflammatory cytokine production in BECs and are inhibited by MaR1. The role of SRF-related signaling in airway epithelial cells is not well characterized, nor has this pathway been previously reported to be modulated by SPM. Therefore, future directions will be aimed at investigating the activation of this pathway in BECs, and how the pathway is modulated by MaR1.

It is noteworthy that $N F \kappa B$ activation was not significantly altered by MaR1 treatment in the cells, as reports have shown that omega-3 fatty acids and their derivatives (including resolvin D1, resolvin D5, and resolvin E1), limit NFKB activation, implicating this transcription factor as a key target of various SPM [14,16,43-45]. The lack of alteration in total $\mathrm{NF} k \mathrm{~B}$ activity with MaR1 treatment indicates diversity in the mechanisms by which the various SPM function and highlights the prospects for

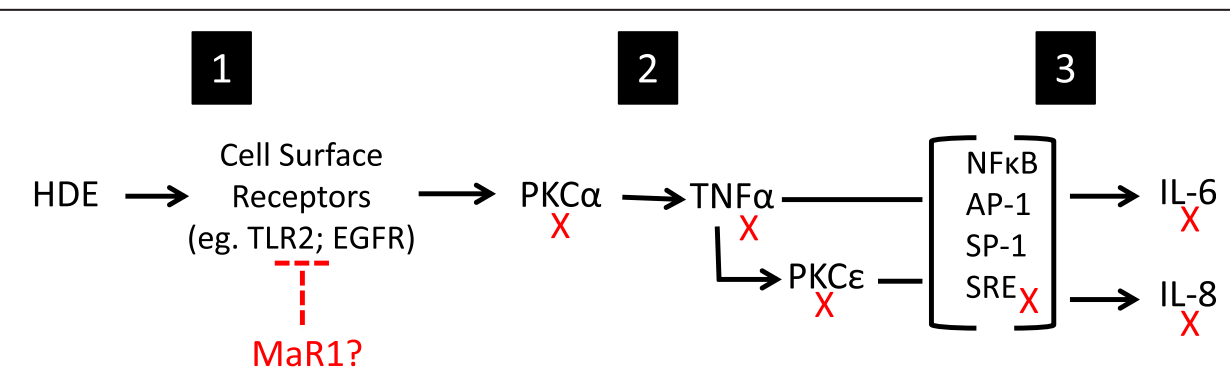

Figure 6 Summary of known and proposed pro-inflammatory signaling events occurring in BECs following HDE exposure. The complex composition of HDE leads to activation at multiple surface receptors, including growth factor receptors (i.e. EGFR) and toll-like receptors (i.e.TLR2) (1) $[35,36]$. HDE causes PKCa activity to increase within 1 hr, leading to TNF-a release and activation of PKC $\varepsilon$ by 6 hours (2) [8]. IL-6 (dependent on TNF-a) and IL-8 (dependent on PKCE) release occurs by 24 hours post-HDE exposure (3) [7,8]. Our data implicate activation of NFKB, AP-1, SP-1, and SRE-related transcription factors in the HDE-induced pro-inflammatory response. Our data reported here indicate that MaR1 reduces PKCa and PKC $\varepsilon$ activities, SRE DNA binding activities, and TNF- $a, I L-6$, and IL-8 release. The direct mechanisms of action of MaR1 on HDE-modulated pathways is undefined but is likely upstream of PKCa activation. Other SPM are known to act through various GPCRs with agonist and antagonist actions, although MaR1 receptor interactions are unknown. A red X indicates a feature of the HDE-induced pro-inflammatory response in $\mathrm{BEC}$ s that $\mathrm{MaR} 1$ reduces. 
tailoring specific SPM to different diseases based on their differing activation signatures. Although, it is important to note that $\mathrm{NF} \kappa \mathrm{B}, \mathrm{AP}-1$, and $\mathrm{SP}-1$ each regulate a substantial number of different gene targets and receive integrative signals to do so [46-48]; it is possible that MaR1 may affect transcriptional activities of specific gene sets regulated by these transcription factors that may cause only subtle changes in total binding activities. Taken together, our data regarding NFkB, AP-1, SP-1, and SRE DNA binding activities suggest MaR1 is acting in part through modulation of SRE-related signaling. Although, we anticipate that MaR1 is also acting through alternate signaling pathways to counterbalance the proinflammatory stimulation propagated through these pathways in HDE-stimulated BECs.

\section{Conclusion}

In conclusion, the experiments described in this report indicate a novel role for MaR1 in HDE-induced BEC inflammatory responses whereby MaR1 reduced PKC activation, resulting in diminished epithelial cell TNF- $\alpha$, IL-6, and IL-8 production following HDE stimulation. DNA binding activity assays revealed MaR1 to have a modulatory effect on HDE-induced SRE-related signaling. Furthermore, studies utilizing the ex vivo mouse lung slice model reveal the potential of MaR1 in preventing inflammation when given either prior to or following HDE exposure. Together, these data provide support for further investigations aimed at determining the potential utility of MaR1 in limiting organic dust-induced inflammation in an in vivo model. A body of work shows that supplementing the diets of patients suffering from acute lung injury or acute respiratory distress syndrome with $\omega-3$ PUFAs significantly lessened lung inflammation and mortality [49]; these studies highlight the potential of SPMs (like MaR1) for treating/ameliorating inflammatory lung conditions. In vivo investigations will therefore be highly relevant for determining the potential utility of MaR1 and other SPM in preventing and/or treating organic dust-related exposures in agriculture workers.

\section{Abbreviations \\ AP-1: Activator protein-1; BEC: Bronchial epithelial cell; CXCL1: C-X-C motif chemokine ligand 1; HDE: Hog confinement facility-derived organic dust extract; IL-6: Interleukin-6; IL-8: Interleukin-8; MAPK: Mitogen-activated protein kinase; MaR1: Maresin-1; NFkB: Nuclear factor kappa-light-chain-enhancer of activated B cells; PKA: Protein kinase A; PKC: Protein kinase C; PMA: Phorbol 12-myristate 13-acetate; SP-1: Specificity protein-1; SPM: Specialized pro- resolving mediators; SRE: Serum response element; SRF: Serum response factor; TNF-a: Tumor necrosis factor-alpha.}

\section{Competing interests}

The authors declare that they have no competing interests.

\section{Authors' contributions}

TMN contributed to the experimental designs and hypotheses involved in the manuscript, carrying out experimental procedures involved in the manuscript, wrote the manuscript, and contributed to the editing and review of the manuscript. AJH contributed to carrying out experimental procedures involved in the manuscript, and contributed to the editing and review of the manuscript. TAW, JAP, TDL, DRC, and DJR contributed to the experimental designs and hypotheses involved in the manuscript, and contributed to the preparation, editing, and review of the manuscript. All authors read and approved the final manuscript.

\section{Authors' information}

Contributing Authors' contact information:

Tara M. Nordgren: 985910 Nebraska Medical Center, Omaha NE 68198 Art J. Heires: 988090 Nebraska Medical Center, Omaha NE 68198 Todd A. Wyatt: 985910 Nebraska Medical Center, Omaha NE 68198 Jill A. Poole: 985300 Nebraska Medical Center, Omaha NE 68198 Tricia D. LeVan: 985910 Nebraska Medical Center, Omaha NE 68198 D. Roselyn Cerutis: 2500 California Plaza, Omaha NE 68178.

\section{Acknowledgements}

This work was supported by NIH-NIOSH (R01OH008539) to DJR, NIH-NIEHS (R01ES019325) to JAP, NIH-NIAAA (R01AA017993) to TAW, and the Central States Center for Agricultural Safety and Health (NIOSH U54OH010162).

\section{Author details}

${ }^{1}$ VA Nebraska-Western lowa Healthcare System, Research Service, Omaha, NE, USA. ${ }^{2}$ Pulmonary, Critical Care, Sleep \& Allergy, University of Nebraska Medical Center, Omaha, NE, USA. ${ }^{3}$ Department of Environmental, Agricultural, and Occupational Health, University of Nebraska Medical Center, Omaha, NE, USA. ${ }^{4}$ Department of Epidemiology, University of Nebraska Medical Center, Omaha, NE, USA. ${ }^{5}$ Department of Oral Biology \& Pharmacology, Creighton University Medical Center, Omaha, NE, USA.

Received: 27 February 2013 Accepted: 8 May 2013

Published: 10 May 2013

\section{References}

1. Eduard W, Pearce N, Douwes J: Chronic bronchitis, COPD, and lung function in farmers: the role of biological agents. Chest 2009, 136(3):716-725.

2. Langley RL: Consequences of respiratory exposures in the farm environment. N C Med J 2011, 72(6):477-480.

3. Poole JA, Romberger DJ: Immunological and inflammatory responses to organic dust in agriculture. Curr Opin Allergy Clin Immunol 2012, 12(2):126-132.

4. Mitchell DC, Schenker MB: Protection against breathing dust: behavior over time in Californian farmers. J Agric Saf Health 2008, 14(2):189-203.

5. Szczyrek M, Krawczyk P, Milanowski J, Jastrzebska I, Zwolak A, Daniluk J: Chronic obstructive pulmonary disease in farmers and agricultural workers - an overview. Ann Agric Environ Med 2011, 18(2):310-313.

6. Poole JA, Wyatt TA, Oldenburg PJ, Elliott MK, West WW, Sisson JH, Von Essen SG, Romberger DJ: Intranasal organic dust exposure-induced airway adaptation response marked by persistent lung inflammation and pathology in mice. Am J Physiol Lung Cell Mol Physiol 2009, 296(6):L1085-95.

7. Romberger DJ, Bodlak V, Von Essen SG, Mathisen T, Wyatt TA: Hog barn dust extract stimulates IL-8 and IL-6 release in human bronchial epithelial cells via PKC activation. J App/ Physio/ 2002, 93(1):289-296.

8. Wyatt TA, Slager RE, Heires AJ, Devasure JM, Vonessen SG, Poole JA, Romberger DJ: Sequential activation of protein kinase $C$ isoforms by organic dust is mediated by tumor necrosis factor. Am J Respir Cell Mol Biol 2010, 42(6):706-715.

9. Weylandt KH, Chiu CY, Gomolka B, Waechter SF, Wiedenmann B: Omega-3 fatty acids and their lipid mediators: towards an understanding of resolvin and protectin formation. Prostaglandins Other Lipid Mediat 2012, 97(3-4):73-82.

10. Singer $P$, Shapiro $H$, Theilla M, Anbar R, Singer J, Cohen J: Antiinflammatory properties of omega-3 fatty acids in critical illness: novel mechanisms and an integrative perspective. Intensive Care Med 2008, 34(9):1580-1592.

11. Serhan $\mathrm{CN}$ : Controlling the resolution of acute inflammation: a new genus of dual anti-inflammatory and proresolving mediators. J Periodontol 2008, 79(8 Suppl):1520-1526. 
12. Bannenberg GL: Therapeutic applicability of anti-inflammatory and proresolving polyunsaturated fatty acid-derived lipid mediators. ScientificWorldJournal 2010, 10:676-712.

13. Chiang N, Fredman G, Backhed F, Oh SF, Vickery T, Schmidt BA, Serhan CN: Infection regulates pro-resolving mediators that lower antibiotic requirements. Nature 2012, 484(7395):524-528.

14. Seki H, Fukunaga K, Arita M, Arai H, Nakanishi H, Taguchi R, Miyasho T, Takamiya R, Asano K, Ishizaka A, Takeda J, Levy BD: The anti-inflammatory and proresolving mediator resolvin E1 protects mice from bacterial pneumonia and acute lung injury. J Immunol 2010, 184(2):836-843.

15. Aoki H, Hisada T, Ishizuka T, Utsugi M, Kawata T, Shimizu Y, Okajima F, Dobashi K, Mori M: Resolvin E1 dampens airway inflammation and hyperresponsiveness in a murine model of asthma. Biochem Biophys Res Commun 2008, 367(2):509-515.

16. Liao Z, Dong J, Wu W, Yang T, Wang T, Guo L, Chen L, Xu D, Wen F: Resolvin D1 attenuates inflammation in lipopolysaccharide-induced acute lung injury through a process involving the PPARgamma/NFkappaB pathway. Respir Res 2012, 13:110.

17. Hsiao HM, Sapinoro RE, Thatcher TH, Croasdell A, Levy EP, Fulton RA, Olsen KC, Pollock SJ, Serhan CN, Phipps RP, Sime PJ: A novel anti-inflammatory and pro-resolving role for resolvin $\mathrm{d} 1$ in acute cigarette smoke-induced lung inflammation. PLoS One 2013, 8(3):e58258.

18. Serhan CN, Yang R, Martinod K, Kasuga K, Pillai PS, Porter TF, Oh SF, Spite M: Maresins: novel macrophage mediators with potent antiinflammatory and proresolving actions. J Exp Med 2009, 206(1):15-23.

19. Poole JA, Alexis NE, Parks C, MacInnes AK, Gentry-Nielsen MJ, Fey PD, Larsson L, Allen-Gipson D, Von Essen SG, Romberger DJ: Repetitive organic dust exposure in vitro impairs macrophage differentiation and function. J Allergy Clin Immunol 2008, 122(2):375-82. 382.e1-4.

20. Poole JA, Dooley GP, Saito R, Burrell AM, Bailey KL, Romberger DJ, Mehaffy J, Reynolds SJ: Muramic acid, endotoxin, 3-hydroxy fatty acids, and ergosterol content explain monocyte and epithelial cell inflammatory responses to agricultural dusts. J Toxicol Environ Health A 2010, 73(10):684-700.

21. Poole JA, Thiele GM, Alexis NE, Burrell AM, Parks C, Romberger DJ: Organic dust exposure alters monocyte-derived dendritic cell differentiation and maturation. Am J Physiol Lung Cell Mol Physiol 2009, 297(4):L767-76.

22. Poole JA, Wyatt TA, Kielian T, Oldenburg P, Gleason AM, Bauer A, Golden G, West WW, Sisson JH, Romberger DJ: Toll-like receptor 2 regulates organic dust-induced airway inflammation. Am J Respir Cell Mol Biol 2011, 45(4):711-719.

23. Poole JA, Gleason AM, Bauer C, West WW, Alexis N, van Rooijen N, Reynolds SJ, Romberger DJ, Kielian TL: CD11c+/CD11b + Cells are Critical for Organic Dust-Elicited Murine Lung Inflammation. Am J Respir Cell Mol Biol 2012, 2012:2012.

24. Palmberg L, Larsson BM, Malmberg P, Larsson K: Induction of IL-8 production in human alveolar macrophages and human bronchial epithelial cells in vitro by swine dust. Thorax 1998, 53(4):260-264.

25. Larsson BM, Palmberg L, Malmberg PO, Larsson K: Effect of exposure to swine dust on levels of IL-8 in airway lavage fluid. Thorax 1997, 52(7):638-642.

26. Wang Z, Larsson $K$, Palmberg L, Malmberg P, Larsson P, Larsson L: Inhalation of swine dust induces cytokine release in the upper and lower airways. Eur Respir J 1997, 10(2):381-387.

27. Perez JF, Sanderson MJ: The frequency of calcium oscillations induced by $5-\mathrm{HT}, \mathrm{ACH}$, and $\mathrm{KCl}$ determine the contraction of smooth muscle cells of intrapulmonary bronchioles. J Gen Physiol 2005, 125(6):535-553.

28. Wyatt TA, Kharbanda KK, McCaskill ML, Tuma DJ, Yanov D, DeVasure J, Sisson $\mathrm{JH}$ : Malondialdehyde-acetaldehyde-adducted protein inhalation causes lung injury. Alcohol 2012, 46(1):51-59.

29. Wyatt TA, Sisson JH: Chronic ethanol downregulates PKA activation and ciliary beating in bovine bronchial epithelial cells. Am J Physiol Lung Cell Mol Physiol 2001, 281(3):L575-81.

30. Wyatt TA, Slager RE, Devasure J, Auvermann BW, Mulhern ML, Von Essen S, Mathisen T, Floreani AA, Romberger DJ: Feedlot dust stimulation of interleukin- 6 and -8 requires protein kinase Cepsilon in human bronchial epithelial cells. Am J Physiol Lung Cell Mol Physiol 2007, 293(5):L1163-70

31. Wyatt TA, Sisson JH, Allen-Gipson DS, McCaskill ML, Boten JA, DeVasure JM, Bailey $\mathrm{KL}$, Poole JA: Co-exposure to cigarette smoke and alcohol decreases airway epithelial cell cilia beating in a protein kinase Cepsilondependent manner. Am J Pathol 2012, 181(2):431-440.

32. Wyatt TA, Sisson JH, Devasure JM, Yanov D, Heires AJ, Romberger DJ: Bidirectional regulation of bronchial epithelial function by PKA and PKC in organic dust-induced airway injury[abstract]. Am. J. Respir. Crit. Care Med. 2010, 181:A4671.

33. Serhan CN, Dalli J, Karamnov S, Choi A, Park CK, Xu ZZ, Ji RR, Zhu M, Petasis NA: Macrophage proresolving mediator maresin 1 stimulates tissue regeneration and controls pain. FASEB J 2012, 26(4):1755-1765.

34. Serhan CN, Krishnamoorthy S, Recchiuti A, Chiang N: Novel antiinflammatory-pro-resolving mediators and their receptors. Curr Top Med Chem 2011, 11(6):629-647.

35. Dodmane PR, Schulte NA, Heires AJ, Band H, Romberger DJ, Toews ML: Airway epithelial epidermal growth factor receptor mediates hogbarn dust-induced cytokine release but not Ca2+ response. Am J Respir Cell Mol Biol 2011, 45(4):882-888.

36. Bailey KL, Poole JA, Mathisen TL, Wyatt TA, Von Essen SG, Romberger DJ: Toll-like receptor 2 is upregulated by hog confinement dust in an IL-6-dependent manner in the airway epithelium. Am J Physiol Lung Cell Mol Physiol 2008, 294(6):L1049-54.

37. Tal TL, Simmons SO, Silbajoris R, Dailey L, Cho SH, Ramabhadran R, Linak W, Reed W, Bromberg PA, Samet JM: Differential transcriptional regulation of IL-8 expression by human airway epithelial cells exposed to diesel exhaust particles. Toxicol Appl Pharmacol 2010, 243(1):46-54.

38. Li M, Zhong X, He Z, Wen M, Li J, Peng X, Liu G, Deng J, Zhang J, Bai J: Effect of erythromycin on cigarette-induced histone deacetylase protein expression and nuclear factor-kappaB activity in human macrophages in vitro. Int Immunopharmacol 2012, 12(4):643-650.

39. Poole JA, Kielian T, Wyatt TA, Gleason AM, Stone J, Palm K, West WW, Romberger DJ: Organic dust augments nucleotide-binding oligomerization domain expression via an NF-\{kappa\}B pathway to negatively regulate inflammatory responses. Am J Physiol Lung Cell Mol Physiol 2011, 301(3):L296-306.

40. Kafoury RM, Kelley J: Ozone enhances diesel exhaust particles (DEP)induced interleukin-8 (IL-8) gene expression in human airway epithelial cells through activation of nuclear factors- kappaB (NF-kappaB) and IL-6 (NF-IL6). Int J Environ Res Public Health 2005, 2(3-4):403-410.

41. Liu HW, Halayko AJ, Fernandes DJ, Harmon GS, McCauley JA, Kocieniewski P, McConville J, Fu Y, Forsythe SM, Kogut P, Bellam S, Dowell M, Churchill J, Lesso H, Kassiri K, Mitchell RW, Hershenson MB, Camoretti-Mercado B, Solway J: The RhoA/Rho kinase pathway regulates nuclear localization of serum response factor. Am J Respir Cell Mol Biol 2003, 29(1):39-47.

42. Soh JW, Lee EH, Prywes R, Weinstein IB: Novel roles of specific isoforms of protein kinase $C$ in activation of the c-fos serum response element. $\mathrm{Mol}$ Cell Biol 1999, 19(2):1313-1324.

43. Recchiuti A, Krishnamoorthy S, Fredman G, Chiang N, Serhan CN: MicroRNAs in resolution of acute inflammation: identification of novel resolvin D1-miRNA circuits. FASEB J 2011, 25(2):544-560.

44. Lo CJ, Chiu KC, Fu M, Lo R, Helton S: Fish oil decreases macrophage tumor necrosis factor gene transcription by altering the NF kappa B activity. J Surg Res 1999, 82(2):216-221.

45. Novak TE, Babcock TA, Jho DH, Helton WS, Espat NJ: NF-kappa B inhibition by omega -3 fatty acids modulates LPS-stimulated macrophage TNFalpha transcription. Am J Physiol Lung Cell Mol Physiol 2003, 284(1):L84-9.

46. Lavrovsky Y, Chatterjee B, Clark RA, Roy AK: Role of redox-regulated transcription factors in inflammation, aging and age-related diseases. Exp Gerontol 2000, 35(5):521-532.

47. Mossman BT, Lounsbury KM, Reddy SP: Oxidants and signaling by mitogen-activated protein kinases in lung epithelium. Am J Respir Cell Mol Biol 2006, 34(6):666-669.

48. Basak S, Hoffmann A: Crosstalk via the NF-kappaB signaling system. Cytokine Growth Factor Rev 2008, 19(3-4):187-197.

49. Pontes-Arruda A, Demichele S, Seth A, Singer P: The use of an inflammation-modulating diet in patients with acute lung injury or acute respiratory distress syndrome: a meta-analysis of outcome data. JPEN J Parenter Enteral Nutr 2008, 32(6):596-605.

doi:10.1186/1465-9921-14-51

Cite this article as: Nordgren et al:: Maresin-1 reduces the proinflammatory response of bronchial epithelial cells to organic dust. Respiratory Research 2013 14:51. 